\title{
Toward Task-Based Mental Models of Human-Robot Teaming: A Bayesian Approach
}

\author{
Michael A. Goodrich and Daqing Yi \\ Brigham Young University, Provo, UT, 84602, USA \\ mike@cs.byu.edu, daqing.yi@byu.edu
}

\begin{abstract}
We consider a set of team-based information tasks, meaning that the team's goals are to choose behaviors that provide or enhance information available to the team. These information tasks occur across a region of space and must be performed for a period of time. We present a Bayesian model for (a) how information flows in the world and (b) how information is altered in the world by the location and perceptions of both humans and robots. Building from this model, we specify the requirements for a robot's computational mental model of the task and the human teammate, including the need to understand where and how the human processes information in the world. The robot can use this mental model to select its behaviors to support the team objective, subject to a set of mission constraints.
\end{abstract}

\section{Introduction}

In complex, rapidly evolving team settings in which a robot fulfills a role, the robot needs sufficient autonomy to allow its human teammates to be free to direct their attention to a wider range of mission-relevant tasks that may or may not involve the robot. In contrast to many prior applications in which the robot was either teleoperated or managed under strictly supervisory control [1], recent advances in robot technologies and autonomy algorithms are making it feasible to consider creating teams in which a robot acts as a teammate rather than a tool [2].

In this team-centered approach, both humans and robots can take on roles that match their strengths. Properly designed, this can facilitate the performance of the entire team. This idea has already been applied to reform human-robot interaction in many areas, like object identification, collaborative tasks performance, etc. [3]. In this paper, we adopt the notion of collaboration, operationally defined as the process of utilizing shared resources (communication, space, time) in the presence of asymmetric goals, asymmetric information, and asymmetric abilities as illustrated in Fig. 11 The word collaboration suggests that there are both overlaps and differences between the goals, information, and abilities of the agents involved. Colloquially, collaboration can happen when everyone has something unique to offer and something unique to gain, but there is some benefit to each individual if activity is correlated.

In a human-robot team, the asymmetries on abilties and information mostly come from the natural difference on agents' sensors and actuators. Additionally, an agent may exhibit ability and information asymmetry in different states of interacting with 


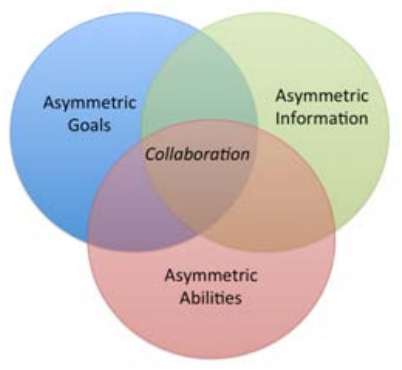

Fig. 1. Operational Elements of Collaboration

the environment, like location, lighting condition etc. Often, a team goal will be decomposed into subgoals in execution. The subgoals are usually assigned to agents in the team by organizing agents into specific roles with specific responsibilities, and this leads to goal asymmetries. In a collaboration framework, the interaction between agents not only focuses on common goals, but may also require providing support for others' goals. In a team search tasks, for example, the robot and the human might work together for target searching, while the robot might assist the human to deal with an emergency.

Collaboration is a form of teamwork that benefits from an explicit representation of shared intent. The theory of shared intent suggests that both the human and the robot need to have a mental model for the task to be performed and another mental model for how other team members will act [4]. The primary contribution of this paper is a framework for developing a task-based mental model from a human-robot collaboration perspective, including the ability to represent and reason about contributions of other team members to the mission and estimation of how other team members' actions affect performance.

\section{Shared Mental Model}

From studies of cognitive psychology, the concept of a shared mental model has been proposed as a hypothetical construct, which has been used to model and explain certain coordinated behaviors of teams. Shared mental models provide a framework of mutual awareness, which serves as the means by which an agent selects actions that are consistent and coordinated with those of its teammates. According to [5] [6], in order to perform collaboratively as a team, members of a team must have the following:

- Teammate Model: knowledge of teammates skills, abilities and tendencies.

- Team Interaction Model: knowledge of roles, responsibilities, information sources, communication channels and role interdependencies.

- Team Task Model: knowledge of procedures, equipment, situations, constraints.

These elements determine (a) how an agent makes decisions as a member of the team and (b) how diverse capabilities and means of interactions are managed within an organizational context. These concepts have been incorporated as important elements in 
existing human-robot team designs [7]. From a robot's perspective, operating within the context of a human-robot team, the robot's shared mental model will help the robot predict information and resource requirements of its teammates. Importantly, a better understanding of task demands and how teammates will likely respond will enhance the robot's ability to support team-level adaptations to changes in the world.

Given a shared intent from the team, the robot is assigned or adopts tasks, either as an autonomous agent or as a collaborating teammate. What does the robot need to collaborate? We address two fundamental elements: (1) How should the robot model the task? (2) How should the robot model a human performing the task? We then illustrate how the concept of a shared mental model is applied within a search task by providing an example computational model that responds to these two questions.

\section{Robot Wingman in a Search Task}

We introduce the shared mental model to a human-robot team search problem. In the problem, the search region is modeled with the belief of where the target objects are, and the search process works as constantly updating this belief by observations. Thus, teams of humans and robots manage a region of space subject to particular time or timing constraints [8].

From prior work in search theory, search efficiency is usually considered as one of the essential factors to a task success, and is therefore a central element of the team's model of a search task. There are several parameters to measure the efficiency in a search task [8], which are determined by the observation capability of a search agent. In this paper, we are interested in:

- Sweep Width: a measure of how wide an area a searcher can, on average, effectively cover. More specifically, it represents how well a sensor (e.g., the human eye) can detect specific objects as a function of distance from sensor to object.

- Coverage: a simple measure of how well a segment was covered by all of the searchers. Coverage is a ratio calculated by summing up the area that each searcher covered and dividing by the area of the search segment.

- Probability of Detection: a measure of the probability of success. Search managers need a way to determine the probability that a lost object would have been found if it was actually in the segment that was searched.

Effective swept width and coverage are determined by the sensor model of a search agent; the sensor model encodes the characteristics and capabilities of the agent's sensors. This model defines what the observation range of an agent is, and how the observation uncertainty might change with the distance of a target object. By contrast, the probability of detection shows the probability that an object would have been detected if in the area, which can be modeled as (a) an agent's prior belief that an object is in the search region and (b) the quality of the agent's observation. Since all agents are imperfect detectors, there exist differences in detection success and detection times among agents.

There is relevant prior work on applying these concepts to human-robot teams. [9] and [10] import robots into urban search and rescue so that human unreachable locations 


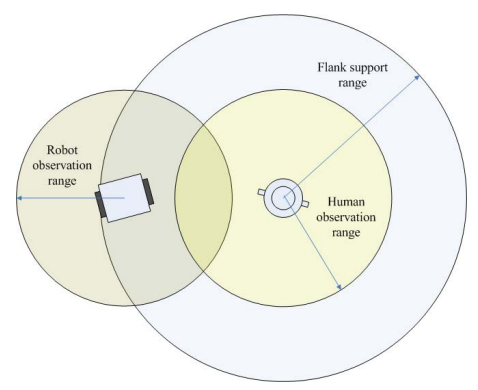

Fig. 2. A Robot Wingman framework

can be explored, which greatly extends the coverage of search task execution. Integrating various types of sensors, like radars, laser rangefinders, ultrasonic sensors etc. [11] [12], greatly expands the sweep width of a search team. [13] and [14] propose a way to improve the probability of detection using information fusion across multiple agents. Modeling the team as a distributed information fusion process exploits the asymmetric perception capabilities of humans and robots to enhance the search efficiency of the team.

In our proposed human-robot search team, we assume that the human is better at strategy and decision making and the robot is better at raw data collection. This assumption forms the basis for the robot's model of its teammate. We propose the notion of a robot wingman to support a human in a collaborative search task, which is to have a robot that accompanies a human as he or she navigates through some space. Since a robot may be able to detect certain types of signals not perceivable by a human (e.g., radio signals or chemical gradients), it is possible for the wingman robot to extend the team's perception not only in space but also in the type of data perceivable by the team. As shown in Fig 2, as a flank support range that constrains where the wingman can move. The robot wingman is expected to stay in an area determined by the flank support range around the human, when the human is moving for the search task. Doing so guarantees that the robot rapidly respond to the human needs assistance, which maintains a reasonable distance for supporting communication and coordination. In a shared human-robot search problem, the robot's role not only includes staying within the flank support range, but also includes gathering information about the world around the team.

The organization of the team determines how information flows when executing the search task. Thus, the organization is an important element of the team interaction model, with information flow acting as the currency of interaction. Information flow shapes the process of fusing asymmetric information for collaboration. In the next section, we model the belief of the locations of the search objects by a shared task model. The information comes from the observations from both the human and the robot. Meanwhile, the robot predicts how the human will work and what the information collected by human is like, and this prediction is used to make a decision on how to run the search operations as in Fig 3 . 


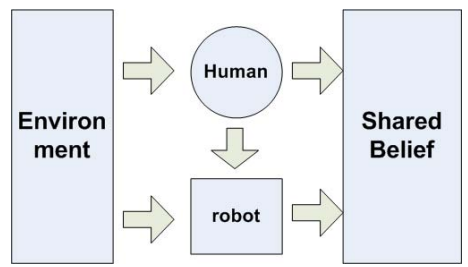

Fig. 3. Information Flow in a Wingman Human-Robot team

\section{A Bayesian Approach}

We present a Bayesian model for how information flows in the world and how information is altered in the world by the locations and perceptions of both humans and robots. Building from this model, we can specify the requirements for a computational mental model of the human teammate to understand where and how the human processes information in the world. The robot can then select its behaviors to support the team objective, subject to a set of mission constraints.

The world is represented as a discrete set of cells. For each cell, we wish to determine the probability that an object of interest is in a particular cell given a set of observations. Let $S_{i}^{t}$ and $O_{i}^{t}$ denote state and observation random variables that encodes whether an object of interest is in cell $i$ at time $t$. Given a set of $N$ cells, we will move or position the robot such that we gather a series of observations that provide information about all of the cells or some subset of those cells.

Since observations will be taken over time and since objects of interest can move over time, we formulate the problem as a sequential Bayes estimation problem. Given $t$ sequential observations about cell $i$, our belief that an object of interest is in cell $i$ at time $t$ is given by the following:

$$
\operatorname{bel}^{t}\left(s_{i}\right)=P_{S_{i}^{t} \mid O_{i}^{t}, O_{i}^{t-1}, \cdots O_{i}^{1}}\left(s_{i}^{t} \mid o_{i}^{t}, o_{i}^{t-1} \cdots o_{i}^{1}\right) .
$$

Equation (1) is the a posteriori estimate that an object of interest in cell $i$ has been detected given all observations to that point position. Adopting the standard conditional independence assumptions of the Bayes filter [15], the sequential estimate becomes

$$
\begin{gathered}
\operatorname{bel}^{t}\left(s_{i}\right)=\alpha P_{O_{i}^{t} \mid S_{i}^{t}}\left(o_{i}^{t} \mid s_{i}\right) \overline{b e l}^{t}\left(s_{i}\right), \\
\overline{b e l}^{t}\left(s_{i}\right)=\sum_{j} \sum_{s_{j} \in S}\left[P_{S_{i}^{t} \mid S_{j}^{t-1}}\left(s_{i} \mid s_{j}\right) b e l^{t-1}\left(s_{j}\right)\right],
\end{gathered}
$$

where $\overline{b e l}^{t}\left(s_{i}\right)$ is the predicted distribution of objects of interest, $\alpha$ is the normalizing constant required by Bayes rule (equal to one divided by the prior predictive distribution), $P_{O_{i}^{t} \mid S_{i}^{t}}\left(o_{i} \mid s_{i}\right)$ is the detection likelihood, and $P_{S_{i}^{t} \mid S_{j}^{t-1}}\left(s_{i} \mid s_{j}\right)$ is the model for how objects move in the world.

In this paper, $s=T$ or $s=F$ indicate that the cell contains an object of interest or not. For each cell, we track the belief that an object of interest is in that cell as a 
function of time. Given a prior belief about objects in the cell, we predict the probability that an object of interest will still be in that cell given (a) the presence or absence of an object in that cell on the previous time step, and (b) the presence or absence of objects in neighboring cells in the previous time step. Thus, Equation (3) includes a double summation, one for all cells in the world (the sum over $j$ ) and the other over the presence or absence of objects in that cell.

The process of a search task can also be considered as information gathering. From (2) and (3), we can see that information from observation updates the belief of the search region, which results in uncertainty reduction. We select entropy, which is a commonly used criterion for measuring uncertainty [16], to quantify information collection. It is written as:

$$
H\left(\operatorname{bel}^{t}\left(s_{i}\right)\right)=-\sum_{s_{i} \in S}\left[\operatorname{bel}^{t}\left(s_{i}\right) \log \left(\operatorname{bel}^{t}\left(s_{i}\right)\right)\right]
$$

\section{Case Study}

Consider a two-dimensional simplified representation of the world and adopt an occupancy grid representation of information in the world. We create a hexagonal tessellation of the world with the dimension of the hexagon determined by the perceptual capabilities of the human. The hexagonal tessellation is useful because it is one in which the distance from the center of one cell to any of its immediate neighbors is constant.

Before exploring the search region, we have no information on this area. We use the entropy of the shared belief to define the uncertainty in equantion (4). More formally, we will assume that the prior probability that a cell is occupied by an object of interest is equal to 0.5, which means that the probabilities of the search object in the cell or not are equivalent.

\subsection{Teammate Model}

From the teammate model of human behavior, the wingman robot can predict how the human will move. This yields a sequence of cells that the human plans to traverse, which is denoted by $Y=\left[y^{1} ; y^{2} ; \cdots y^{D}\right]$. Each $y^{t}$ corresponds to a physical location in the tessellation, so $y^{t}=i$ means that the human was in cell $i$ at time $t$.

We adopt a very simple model of agent perception, albeit one based in search theory. The model is that the likelihood of detecting an object of interest in cell $i$ is certain if the human occupies that cell, is zero for cells outside a fixed radius of detection, and is constant for all cells within the radius of detection. Let $N(i)$ denote the set of all cells that are within $R$ units of cell $i$, in which $R$ defines a radius,

$$
N(i)=\{j: j \text { is no further than } R \text { cells from } i\} .
$$

Let $\lambda \in(0,1)$ be the constant of detection for all cells within $N(i)$. Thus, an agent's probability of detection at position $x^{t}$ is given by Equation (6).

$$
P_{O_{i}^{t} \mid S_{i}^{t}}(F \mid T)=\left\{\begin{array}{lc}
0 & \text { if } i=x^{t} \\
1-\lambda & \text { if } i \in N\left(x^{t}\right) \\
1 & \text { otherwise }
\end{array}\right.
$$


By definition, $P_{O_{i}^{t} \mid S_{i}^{t}}(T \mid T)=1-P_{O_{i}^{t} \mid S_{i}^{t}}(F \mid T)$. In (6), we assume a search agent can do perfect observation in the cell he is in. However, there exist distinctions on probabilities of detection in the neighbor cells, which come from the difference on agent perception capabilities. To differentiate the observation range, we use $N^{h u m a n}\left(y^{t}\right)$ and $N^{\text {robot }}\left(x^{t}\right)$ for the set of observed cells by human and robot at time $t$.

\subsection{Team Interaction Model}

The team interaction model uses the flank support range $R_{\text {flank }}^{\text {human }}$ to determine the set of cells for the wingman robot motion. This is based on the human's tolerance for how far the robot can wander before being out of position. We use (5) to translate $R_{\text {flank }}^{\text {human }}$ into a set of feasible cells, $N_{\text {flank }}^{\text {human }}\left(y^{t}\right)$, in which $y^{t}$ is the human's position. Given a motion range of the wingman robot at a time step, $R_{\text {motion }}^{\text {robot }}$, we have

$$
\forall y^{t}, x_{t} \in N_{\text {flank }}^{\text {human }}\left(y^{t}\right) \cap N_{\text {motion }}^{\text {robot }}\left(x^{t-1}\right)
$$

to define the wingman robot motion dependence on the human motion.

In this paper, we assume that the teammate model provides enough information to estimate the human's path via prediction, Equation (6) can be used to determine the posterior probability of likely target location after the human has moved. The posterior from the human is then used as the prior for the robot. In essence, this means that the shared belief about the world passes through two phases: a refinement that comes because the human has moved through the environment and a refinement that comes because the robot is going to move through the environment.

\subsection{Team Task Model}

When the robot plans to fulfill its role for the task model, it assumes that objects of interest do not move, appear, or disappear over time, but this will change in future work. Given this assumption, the prior estimate for the target object's location at time $t$ is equal to the posterior estimate for target object location at time $t-1$. In future work, if the object of interest can move, then a predictive step is required and a full Bayes filter can be applied [15].

Since the human's path has been obtained from the teammate model (the robot is supporting the human), our goal is to control the robot's path to maximize the amount of information gathered by the human and robot combined. When the robot is at $x^{t}$, it will update the beliefs of all the neighbor cells defined by the radius of detection, $R^{\text {robot }}$. We denote the information gain at position $x^{t}$ as

$$
F\left(x^{t}\right)=\sum_{i \in N^{\text {agent }}\left(x^{t}\right) \cup x^{t}}\left[H\left(\text { bel }_{i}^{t-1}\right)-H\left(\text { bel }_{i}^{t}\right)\right] .
$$

In (8), $H\left(\right.$ bel $\left._{i}^{t}\right)$ denotes the entropy of the belief of cell $i$ at time $t$, and $F\left(x^{t}\right)$ shows the uncertainty reduction at time $t$ from the all the observed cells.

In order to keep synchronization with human motion, a requirement imposed by the interaction and task model, we assume that the robot starts in the same location 


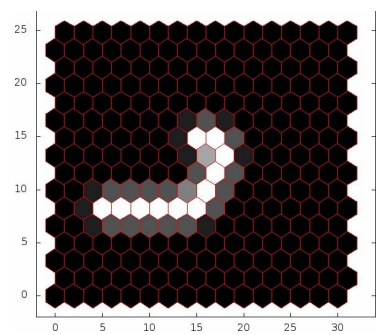

(a) After Human Visited

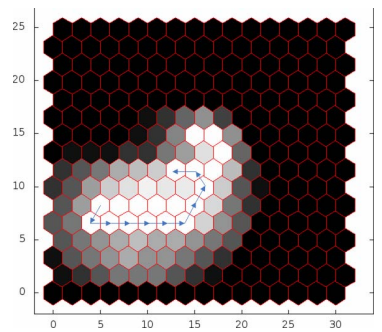

(c) After Wingman Robot Visited, $F S R=2, R O R=3$

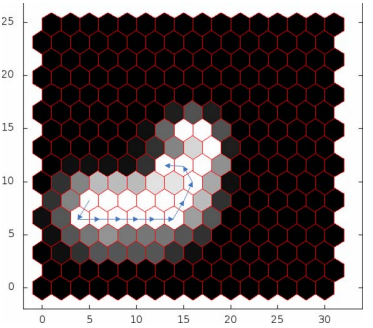

(b) After Wingman Robot Visited, $F S R=2, R O R=2$

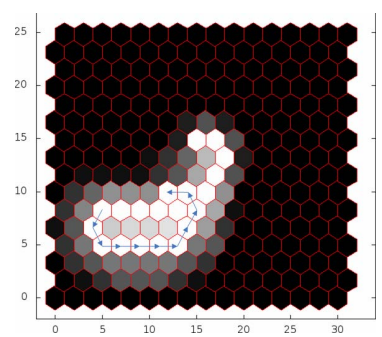

(d) After Wingman Robot Visited, $F S R=3, R O R=2$

Fig. 4. The entropy of shared belief of the search region changed after observation, FSR is short for FlankSupportRange, ROR is short for RobotObservationRange

as the human and intends to plan a time length identical with the predicted human motion length. We set the initial position by $x_{0}=y_{0}$ and the planning time length to $D$. These constraints yield a natural tree structure for the problem, which forms a tree by finding all "visitable" cells by (7). The problem is thus to find the sequence $\mathbf{X}=\left[x^{1} ; x^{2} ; \cdots x^{D}\right]$ of robot positions such that

$$
\sum_{t=1}^{D} F\left(x^{t}\right)=H\left(b e l^{D}\right)-H\left(b e l^{0}\right)
$$

is as large as possible. Performance can either be described as a summation of information gain at each time step or the total information gain reward. Putting this together with the constraint (7) from the team interaction model yields the constrained optimization problem for the team task model.

$$
\begin{gathered}
\max _{x^{1} \ldots x^{D}} \sum_{t=1}^{D} F\left(x^{t}\right) \\
\text { subject to } x^{t} \in N_{\text {flank }}^{\text {human }}\left(y^{t}\right) \cap N_{\text {motion }}^{\text {robot }}\left(x^{t-1}\right) .
\end{gathered}
$$

Fig 4 shows a case of how the entropy of the shared belief of the search region has been updated by the search of the human-robot team. To visualize the entropy, we color the maximum value in black and minimum value in white, which determines the gray transition for the values in between. Before the search begins, we assume we have no 
idea on the location of the search object so that all the cells have been colored black which shows the largest uncertainty. For visited cells, the entropy is reduced to zero. This means the entropy of the shared belief of this cell has been reduced to zero so that this cell has been colored white.

\subsection{Simulation}

Fig 4(a) shows how the entropy of the shared belief of the search region has been changed by a human search agent. The robot's teammate model assumes, via Equation (6), that the human has imperfect observation in neighboring cells, so the entropy of the believes on neighboring cells has been reduced less than the visited cells. The value of the gray color is determined by how the observation model is defined.

Based on the human path and how the shared belief of the environment has changed, the robot wingman plans a path to optimize the team information gain, subject to the teammate interaction model via Equation (7). Fig 4(b) 4(c) and 4(d) show the entropy of the shared belief of the search region after the search of the robot wingman within different parameters. We use arrows to label the planned path of the robot wingman. We can see that increasing the observation range of the robot will usually not influence the planned path for the robot wingman, as Fig 4(b) and 4(c) have the same path shapes. However, increasing the flank support range, which gives more motion freedom to the robot wingman, will lead to a new generated path, as shown in Fig 4(d).

\section{Conclusion and Future Work}

Based on shared mental model and search theory, we model team-based search as an information-based task using a Bayesian approach. A wingman robot has been introduced for this problem, with robot decision algorithms designed to support collaborative human-robot interaction. Using the entropy of the belief of the search region as a way of information measurement, we illustrate how the robot wingman will do path planning for collaborating with the human as an optimization problem. Using a specific case, we illustrate how the human robot collaboration on a search task will change the entropy of the belief of the search region, which works as a shared model on the environment from the team perspective. Here we only use a depth-first exhaustive search to find the optimal solution for wingman path planning. Future work will be focused on proposing an efficient and applicable solution for wingman path planning. Moreover, we will add more features on modeling the search environment, like obstacles and stochastic dynamics. Finally, we will relate problem modeling assumptions to the requirement of a shared mental model.

Acknowledgments. We would like to thank the U.S. Army Research Lab for providing funding for this work. The views of this paper do not necessarily reflect the views of the funding agency. 


\section{References}

[1] Parasuraman, R., Sheridan, T., Wickens, C.: A model for types and levels of human interaction with automation. IEEE Transactions on Systems, Man and Cybernetics, Part A: Systems and Humans 30(3), 286-297 (2000)

[2] Breazeal, C., Gray, J., Hoffman, G., Berlin, M.: Social robots: beyond tools to partners. In: 13th IEEE International Workshop on Robot and Human Interactive Communication, ROMAN 2004, pp. 551-556 (September 2004)

[3] Hoffman, G., Breazeal, C.: Collaboration in human-robot teams. In: Proc. of the AIAA 1st Intelligent Systems Technical Conference, Chicago, IL, USA (2004)

[4] Salas, E., Fiore, S., Letsky, M.: Theories of team cognition: Cross-disciplinary perspectives. Routledge Academic (2011)

[5] Mathieu, J.E., Heffner, T.S., Goodwin, G.F., Salas, E., Cannon-Bowers, J.A.: The influence of shared mental models on team process and performance. Journal of Applied Psychology 85(2), 273 (2000)

[6] Fiore, S.: Personal communication

[7] Neerincx, M., de Greef, T., Smets, N., Sam, M.: Shared mental models of distributed human-robot teams for coordinated disaster responses. In: 2011 AAAI Fall Symposium Series (2011)

[8] Roscheck, M., Goodrich, M.: Detection likelihood maps for wilderness search and rescue. In: 2012 IEEE International Conference on Systems, Man, and Cybernetics (SMC), pp. 327-332. IEEE (2012)

[9] Casper, J., Murphy, R.: Human-robot interactions during the robot-assisted urban search and rescue response at the world trade center. IEEE Transactions on Systems, Man, and Cybernetics, Part B: Cybernetics 33(3), 367-385 (2003)

[10] Doroodgar, B., Ficocelli, M., Mobedi, B., Nejat, G.: The search for survivors: Cooperative human-robot interaction in search and rescue environments using semi-autonomous robots. In: 2010 IEEE International Conference on Robotics and Automation (ICRA), pp. 2858-2863 (May 2010)

[11] Ruangpayoongsak, N., Roth, H., Chudoba, J.: Mobile robots for search and rescue. In: 2005 IEEE International Safety, Security and Rescue Robotics, Workshop, pp. 212-217. IEEE (2005)

[12] Burion, S.: Human detection for robotic urban search and rescue. Diploma Work (2004)

[13] Nourbakhsh, I.R., Sycara, K., Koes, M., Yong, M., Lewis, M., Burion, S.: Human-robot teaming for search and rescue. IEEE Pervasive Computing 4(1), $72-79$ (2005)

[14] Burke, J., Murphy, R.: Human-robot interaction in usar technical search: two heads are better than one. In: 13th IEEE International Workshop on Robot and Human Interactive Communication, ROMAN 2004, pp. 307-312 (September 2004)

[15] Thrun, S., Burgard, W., Fox, D., et al.: Probabilistic robotics, vol. 1. MIT Press, Cambridge (2005)

[16] Cover, T., Thomas, J.: Elements of information theory. Wiley Interscience (2006) 